\title{
Face Recognition with Irregular Region Spin Images
}

\author{
Yang Li, William A.P. Smith, and Edwin R. Hancock \\ Department of Computer Science, University of York, York, YO10 5DD, UK
}

\begin{abstract}
This paper explores how spin images can be constructed using shapefrom-shading information and used for the purpose of face recognition. We commence by extracting needle maps from gray-scale images of faces, using a mean needle map to enforce the correct pattern of facial convexity and concavity. Spin images [6] are estimated from the needle maps using local spherical geometry to approximate the facial surface. Our representation is based on spin image histograms for an arrangement of image patches. Comparing to our previous spin image approach, the current one has two basic difference: Euclidean distance is replaced by geodesic distance; Irregular face region is applied to better fit face contour. We demonstrate how this representation can be used to perform face recognition across different subjects and illumination conditions. Experiments show the method to be reliable and accurate, and the recognition precision reaches 93\% on CMU PIE sub-database.
\end{abstract}

\section{Introduction}

Face recognition is an active research area that has been approached in many ways. Roughly speaking the existing methods can be divided in two categories. The first is the feature-based method, while the second is the model-based method. Recently, it is the model-based methods that have attracted the greatest attention [2]. Here one of the most important recent developments is the work of Blanz and Vetter [3]. In this work a 3D morphable model is matched to face data using correspondences delivered by optic flow information. The method gives recognition rates of about $80 \%$ when profiles are used to recognise frontal poses. However, the construction of the model requires manual marking feature points, which is labour intensive. Hence, the automatic construction of models remains an imperative in face recognition. There are related feature-based approaches which are based on the assumption that face images are the result of Lambertian reflectance. Under this assumption 3D linear subspaces can be constructed that account for facial appearance under fixed viewpoint but under different illumination [12 1 10].

In this paper we aim to develop a feature-based method for face recognition that can be used to recognise faces using surface shape information inferred from image brightness using a Lambertian shape-from-shading scheme. Shape-from-shading is not widely accepted as a technique for face recognition. The reason is that surface normal is commonly believed to be noisy and is unstable under changes in illumination direction or change of pose. However, recently it has been shown that shape-from-shading can be used to extract useful features from real world face images [11].

One of the problems that hinders the extraction of reliable facial topography using shape-from-shading is the concave/convex inversions that arise due to the bas-relief

B.K. Ersbøll and K.S. Pedersen (Eds.): SCIA 2007, LNCS 4522, pp. 730 739, 2007.

(C) Springer-Verlag Berlin Heidelberg 2007 
ambiguity. A recent paper [11] have shown how this problem can be overcome using a statistical model for admissible surface normal variations trained on range data. Here we use a simplified version of this algorithm. The surface normals are constrained to fall on the Lambertian reflectance with axis in the light source direction and apex angle given by the inverse cosine of the normalised image brightness. The position of the surface normal on the cone is decided to minimize its distance to the corresponding mean surface normal direction.

To construct a surface representation from the surface normals, we turn to the spin image first developed by Johnson and Hebert [6]. A spin image is a group of histograms constructed from the polar coordinates of arbitrary reference points on a surface. The representation can capture fine topographic surface details. Unfortunately, the computational overheads associated with the method are high, since a histogram needs to be generated for each surface location. Moreover, the original spin image representation was developed for range images and hence relies on surface height rather than surface normal information. We demonstrate how these two problems can be overcome by computing local spin images on image patches using surface normal information.

\section{Mean Needle Map Alignment}

The shape-from-shading algorithm used to extract needle maps from brightness images is as follows. We follow the work in [13] and place the surface normal on a cone whose axis is the light source direction and whose opening angle is the inverse cosine of the normalised image brightness.

This initial surface normals typically contains errors, and in particular locations where the pattern of convexity or concavity is reversed. To overcome this problem we draw on a model that accounts for the distribution of surface normals across groundtruth facial surfaces. To construct this model we use a sample of range images of human faces. From the gradients of surface height data, we make estimates of surface normal direction. The resulting fields of surface normals are adjusted so that faces have the same overall centering, scale and orientation. At each location we compute the mean surface normal direction according to the available set of ground-truth surface normals. Here we use the Max-Planck database which has 200 sample images of male and female subjects.

We use the mean facial needle map to adjust the positions of the surface normals on the reflectance cones. Each initial surface normal is rotated on its cone so that it minimises the angle subtended with the mean surface normal at the corresponding image location.

$$
f(x, y)=\operatorname{argmin}\left(\left|\theta_{r}(x, y)-\theta_{\text {mean }}(x, y)\right|\right)
$$

where $\theta_{r}$ and $\theta_{\text {mean }}$ are the azimuth angles of the aligned surface normal $n_{r}$ and the mean surface normal $n_{\text {mean }}$ on the surface point $(x, y)$.

The simplest way to satisfy Eqn.11 is to make sure the aligned surface normal $n_{r}$, the mean surface normal $n_{\text {mean }}$, and the illumination cone axis $n_{x}$ are on the same plane.

In Fig. 3 we illustrate the improvements gained using this simple shape-from-shading procedure. In the top row of the figure we show the input images of a single subject with the light source in different directions. In the second row we show the initial estimates 
of the surface normal directions. Here we have visualised the needle maps by taking the inner product of the surface normal with the light-source vector perpendicular to the image plane. This is equivalent to re-illuminating the surface normals with frontal Lambertian reflectance. From the images in the second row it is clear that there are significant concave/convex inversions in the proximity of the nose and lips when the face is illuminated obliquely. In the third row of the figure we show the surface normals that result from the adjustment procedure described above. The re-illuminations reveal that the inversions are removed and the quality of the recovered facial topography is improved (Fig. 4 illustrates the solution of this inversion problem).

\section{The Original Spin Image Approach}

The spin image of Johnson and Hebert [6] aims to construct an object-centered representation. The representation consists of a group of histograms and is constructed in the following manner: Commence by selecting an arbitrary point on the surface as the reference point $O$, and $\overrightarrow{n_{O}}$ is the surface normal at the point $O$. Then select a second arbitrary point $P$ on the surface, and $\overrightarrow{n_{p}}$ is the surface normal at the point $P$. Assume the object resides in a $3 \mathrm{D}$ coordinate system with the surface normal $\overrightarrow{n_{o}}$ as $z$ axis and the $x y$ plane perpendicular to $\overrightarrow{n_{o}}$. The Euclidean distance $\gamma=|\overrightarrow{O P}|$ can be projected onto the $x y$ plane as $\alpha$ and the $z$ axis as $\beta$ respectively. After the distances $\alpha$ and $\beta$ of all the surface points are calculated, we can use them to construct a histogram. The above procedure is performed after each point on the surface has been taken as the point $P$ so that a single histogram is constructed, and then a group of histograms are constructed using the above steps and taking each point on the surface as the reference point $O$. Figure 1 illustrates the spin image construction.

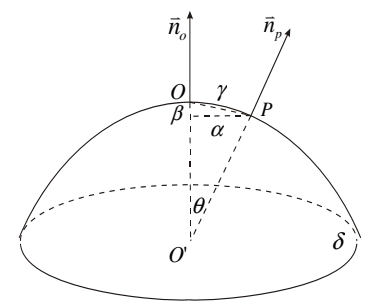

Fig. 1. Illustration of spin image construction

This object-centered representation is invariant to scale, translation and rotation since the spin image is calculated using only relative distances between object surface points.

The spin image representation is based on the availability of surface height data and can not be applied directly to fields of surface normals or needle maps. Moreover, spin image histograms need to be constructed at each image location, and this is demanding in both computation time and storage. In order to obtain this object-centered representation for an object with $n$ surface points/image pixels, the computation cost will be $O\left(n^{2}\right)$. 


\section{Patch-Based Spin Images Adaptation}

We have adapted a patch-based approach to spin image representation. We segment surface into patches and for each patch we use only the geometric center point $O$ as reference point to construct spin image, rather than use every point of this surface as in the original spin image approach. Our histograms are constructed on a patch-by-patch basis.

\subsection{Region Segmentation}

As we mentioned before, we will develop patch-based spin image representation here. In our previous approach [7], a primitive fixed rectangle segmentation strategy was employed to obtain face patches. From Fig. 2, we can clearly see that the major problem in this strategy is the patches dos not represent natural face components, which makes different faces less distinguishable. Also some patches include the unnecessary background, which might involve non-face information if the face is with clutter background.

In this paper, we will propose a more sophisticate way for patch segmentation, which employs Active Appearance Models(AAM) [4]. It is a statistical model of shape and grey-level appearance. After proper training, it can locate the face feature points, and we can use this information to construct our irregular patches.

We first divide face and background into rectangular patches using our previous fixed rectangle segmentation strategy [7]. We manually preset our feature points on distinguishing face contour points, e.g. eye corners, brow outline points, etc. Then we train AAM to obtain feature points on all subject faces. Search for the overlapped parts of the rectangular patches and the polygonal area within the face contour, we can effectively exclude the background. Because the surface normal works poorly on the face parts of eyes/eyebrows/hair, we also need to exclude those face parts from the face region.

From Fig. 2. we can see that after combing the rectangular patch segmentation and the feature point location, the patches only contain face regions but not the background. Also the unnecessary parts are excluded from the patch. So the spin image can be constructed on more distinguishable face patches.
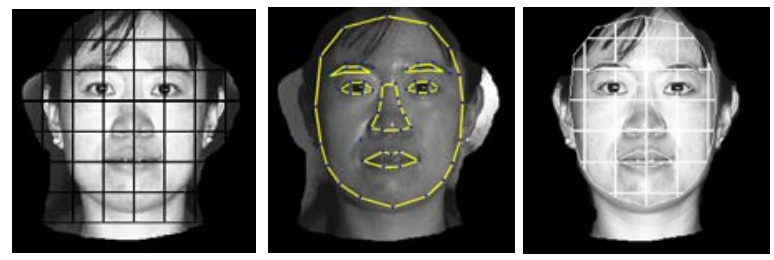

Fig. 2. This figure illustrates the original rectangular patch segmentation, the feature point location generated by AAM and the irregular patch segmentation 


\subsection{From Surface Normal to Spin Image Histogram}

From the GGFI [9] we can obtain a surface height map $\Phi_{H M}$ over the surface, and the relative height $\beta_{O P}$ between any two surface points $O$ and $P$ in the viewing direction $\overrightarrow{n_{v}}$ can be obtained from the height map $\Phi_{H M}$.

We used to use the Euclidean distance $\alpha_{O P}$ between $O$ and $P$ perpendicular to the viewing direction $\overrightarrow{n_{v}}$ [7], but since the mesh-modeling can represent small regions of face surface more naturally than smooth planes or sphere regions, now we switch to the geodesic distance.

The geodesic distance $\gamma_{P_{1} P_{2}}$ between two neighbour points $P_{1}$ and $P_{2}$ is defined as follows:

$$
\gamma_{P_{1} P_{2}}=\sqrt{\alpha_{P_{1} P_{2}}^{2}+\beta_{P_{1} P_{2}}^{2}}
$$

where $\alpha_{P_{1} P_{2}}$ is the distance on the image plane, which is perpendicular to the viewing direction $\overrightarrow{n_{v}} ; \beta_{P_{1} P_{2}}$ is the relative height between the points $P_{1}$ and $P_{2}$ in the viewing direction $\overrightarrow{n_{v}}$, which can be acquired from in the height map $\Phi_{H M}$ as mentioned before in this section.

The geodesic distance $\gamma_{P_{1} P_{n}}$ between two points $P_{1}$ and $P_{n}$ connected by the path path $_{P_{1} P_{n}}$ is defined as follows:

$$
\gamma_{P_{1} P_{n}}=\sum_{i=1}^{n-1} \gamma_{P_{i} P_{i+1}}
$$

where $P_{1}, P_{2}, \ldots, P_{n}$ is the neighbour points along the path path $h_{P_{1} P_{n}}$.

Assume there is $N$ paths from the point $O$ to the point $P$, the geodesic distance $\beta_{O P}$ between $O$ and $P$ should be the shortest distance among these paths on the meshmodeling.

$$
\beta_{O P}=\min \left\{\operatorname{path}_{O P}^{1}, \operatorname{path}_{O P}^{2}, \ldots, \operatorname{path}_{O P}^{N}\right\}
$$

Our solution to this shortest path problem is implemented by the following procedure (Dijkstra's algorithm) [5].

We now have all the ingredients to construct the histogram of $\beta$ and $\gamma$ for the surface patch centered at the point $O$.

In our experiment we construct a 10 by 10 bin histograms of $\beta$ and $\gamma$ for an image patch less than equal to 32 by 32 pixels. The exact pixel number depends on how many of them fall into the face region. The histogram is also normalised so as to be scale invariant.

As an additional step, we have performed PCA on spin image histograms to reduce data dimensionality. We normalise the bin contents of each spin image histogram to unity. The normalised bin contents of histograms are concatenated as a long-vector as follows: 


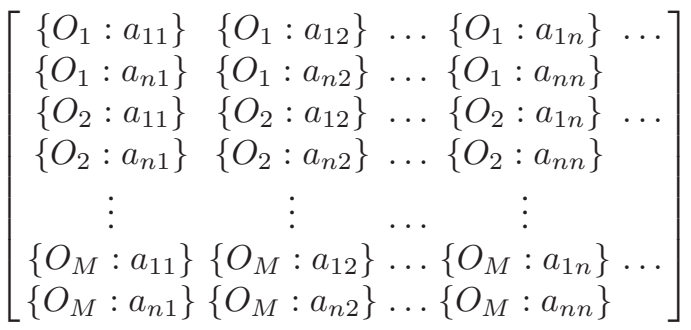

where $\left\{O_{1}, \ldots, O_{M}\right\}$ are spin image histograms, and $\left\{O_{i}: a_{j k}\right\}$ is the $(j, k)$ bin of the $i$ th histogram.

Dimensionality reduction is effected by projecting the long-vector onto the leading eigenvectors of the long-vector covariance matrix.

In the adaptation of spin image on surface normal, the computation cost is reduced to $O(n)$ instead of $O\left(n^{2}\right)$ in the original approach.

\section{Recognition}

In our preprocessing of the images to extract needle maps, we perform alignment. This means that we can apply a patch template to the extracted needle maps to decompose the face into regions. The patch template is constructed from the mean facial needle map, and consists of regions that are either wholly concave or wholly convex. The convexity/concavity test is made using the sign of the changes in surface normal direction. By performing the spin image analysis on these regions, we avoid problems associated with inflexion points when the approximations outlined in Sect. 2] are employed.

As an alternative to constructing the template from the mean needle map, we have explored constructing it from the needle map extracted from each facial image.

Our measure of facial similarity is based on the normalised correlation of the spin image histograms for corresponding template patches.

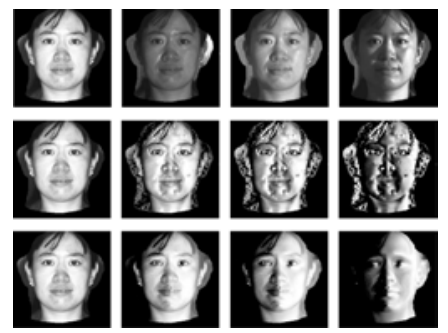

Fig. 3. The images in the first row are real images illuminated by the light sources from different directions. The images in the second/third row are the original needle maps[13]/the needle maps treated with Mean Needle Map Alignment (Sect. 2] respectively rendered by the frontal light source. The images in the third row are more photo-realistic and carry less noise than the ones in the second row. 

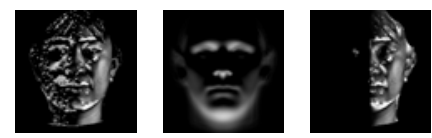

Fig. 4. The first image is the original needle map projected to the image plane. The second image is the mean needle map that we use as the template. The third image is the needle map projected to the image plane after the Mean Needle Map Alignment (Sect.22). The third image compensate the concave/convex problem of the first one.

Johnson and Hebert use normalised correlation to evaluate spin image similarity [6]. The method assumes that spin images from proximal points on the surface for different views of an object will be linearly related. This is because the number of points that fall into corresponding bins will be similar (given that the distribution of points over the surface of the objects is the same). In our case, this assumption still holds. We hence use normalised correlation to compare the patch-based spin images. The correlation between two single patch spin image histograms $x$ and $y$ of different spin images $X$ and $Y$ is given by

$$
r_{x y}=\frac{n \sum x_{i} y_{i}-\sum x_{i} \sum y_{i}}{\sqrt{\left(n \sum x_{i}^{2}-\left(\sum x_{i}\right)^{2}\right)\left(n \sum y_{i}^{2}-\left(\sum y_{i}\right)^{2}\right)}}
$$

where $r_{x y}$ is the correlation of two histograms $x$ and $y . n$ is the bin number of histograms, $x_{i}$ and $y_{i}$ are the $i$ th bin contents of two histograms $x$ and $y$ respectively.

The correlation between two spin images $X$ and $Y$ is given by

$$
r_{\text {sum }}=\sum_{i=1}^{\min (M, N)} r_{m_{i} n_{i}}
$$

where $M$ and $N$ are the number of histograms of two spin images $X$ and $Y$, and $m_{i}$ and $n_{i}$ are the $i$ th histograms $x$ and $y$ of two spin images $X$ and $Y$ respectively.

\section{Experiments}

We apply our method to the CMU PIE face database. We use only frontal-viewed face images in this paper. The sub-database contains $67 \times 7=469$ (67 subjects (1-67) and 7 lights $(3,10,7,8,9,13,16))$ images. We apply the two different patch segmentation strategies outlined above.

For the 7 images of the same subject illuminated by different lights, we use 3 of them for the training set and 4 of them for the test set. To perform recognition on the 67 subjects, we select a probe image from the training set and order the images in the test sets according to their similarities. The results of our experiments are summarised using the precision-recall curves shown in Fig. 5.

The plus-dotted curve shows the result of using a global histogram of curvature attributes extracted from the needle maps [8]. The circle-dotted curve shows the result of our rectangular patch spin image, and the star-dotted curve shows the corresponding 
spin image vector. The cross-dotted curve shows the result of our new irregular patch spin image, and the square-dotted curve shows the corresponding spin image vector. The irregular patch spin image vector gives the best performance.

In Table 1 we show the result of applying the various shape representations to the initial needle maps and the needle maps adjusted with Mean Needle Map Alignment (MNMA). In each case there is a significant improvement, and therefor in the following experiments we only use the needle maps adjusted with MNMA.

Table 1. Recognition rates using the initiate surface normal and the one adjusted with MNMA

\begin{tabular}{l|c|c}
\hline & Original & MNMA \\
\hline Global Histogram & $37.50 \%$ & $47.91 \%$ \\
\hline Irregular Spin Image & $75.23 \%$ & $92.61 \%$ \\
\hline Irregular Vector & $78.10 \%$ & $92.99 \%$ \\
\hline
\end{tabular}

Table 2. Recognition rates obtained by the rectangular/irregular patch spin image and the corresponding PCA spin image vector. The performances of PCA vector are slightly better.

\begin{tabular}{l|c|c|c|c}
\hline & Rectangular Spin Image & Rectangular Vector & Irregular Spin Image & Irregular Vector \\
\hline Face Components & $27.81 \%$ & $29.69 \%$ & $30.42 \%$ & $32.71 \%$ \\
\hline Whole Face & $80.67 \%$ & $81.57 \%$ & $92.61 \%$ & $92.99 \%$ \\
\hline
\end{tabular}

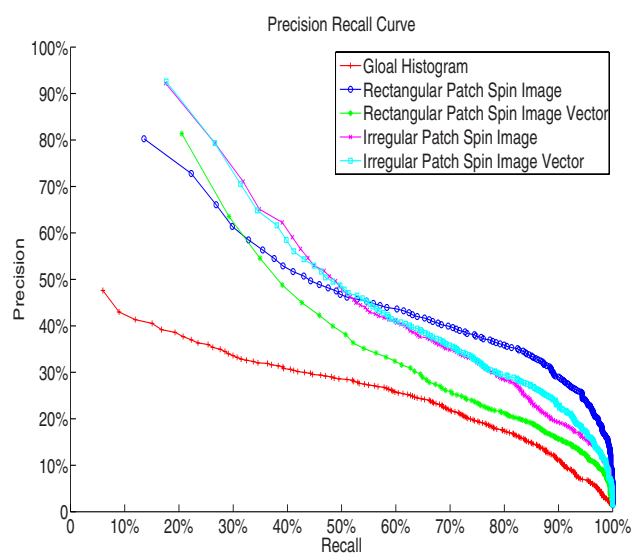

Fig. 5. There are five precision-recall curves of different approaches in this figure: the global histogram approach and the rectangular/irregular patch spin image/vector approaches. All these results are based on the surface normal processed by the Mean Needle Map Alignment because that approach has been proved improving the distinguishing ability in Table 1 Among them the irregular patch spin image vector approach gives the best performance.

In Table 2 we compare the recognition results obtained using the spin image and applying PCA to the spin image long-vectors. Performance is improved using PCA, 
and this can be ascribed to the fact that PCA effectively discards the histogram bins that are associated with insignificant variance.

Please notice the face component performance is obtained by only comparing the similarity of a single face component (cheek, nose, mouth, etc.) instead of the whole face, so the recognition rate will be reasonably low and can only be used to compare the performance of these four methods.

\section{Conclusion and Future Work}

In this paper we have proposed a more advanced approach comparing to our previous one [7] to extract spin images from 2D facial images using shape-from-shading. We made a few changes. First, we use geodesic distance to replace Euclidean distance in spin images to better describe the 3D shape. Second, we use the irregular patch to replace the rectangular patch to build spin images to exclude the background and unwanted face parts so that spin images can involve less surface normal errors. These make the irregular patch spin image obtain better result than the previous approach.

In the next step, we will apply this approach to faces with various poses to check its performance under more difficult circumstances, and also we will look for better way of similarity evaluation by adding weight for each histogram in spin image according to their locations or sizes.

\section{References}

1. Belhumeur, P., Kriegman, D.: What is the set of images of an object under all possible illumination conditions? International Journal of Computer Vision 28(3), 245-260 (1998)

2. Blanz,: Automatic face identification system using flexible appearance models. IVC 13(5), 393-401 (1995)

3. Blanz, V., Vetter, T.: Face recognition based on fitting a 3d morphable model. IEEE Transactions on Pattern Analysis and Machine Intelligence 25(9), 1063-1074 (2003)

4. Cootes, T.F., Edwards, G.J., Taylor, C.J.: Active appearance models. In: Burkhardt, H., Neumann, B. (eds.) ECCV 1998. LNCS, vol. 1407, pp. 484-498. Springer, Heidelberg (1998)

5. Dijkstra, E.W.: A note on two problems in connexion with graphs. In: Numerische Mathematik, vol. 1, pp. 269-271. Mathematisch Centrum, Amsterdam, The Netherlands (1959)

6. Johnson, A.E., Hebert, M.: Using spin images for efficient object recognition in cluttered 3d scenes. IEEE Transactions on Pattern Analysis and Machine Intelligence 21(5), 433-449 (1999)

7. Li, Y., Hancock, E.: Face recognition using shading-based curvature attributes. In: International Conference on Pattern Recognition ICPR, Cambridge, UK (August 2004)

8. Li, Y., Hancock, E.: Face recognition using shading-based curvature attributes. In: International Conference on Pattern Recognition ICPR, Cambridge, UK (August 2004)

9. Frankot, R.T., Chellappa, Z.: A method for enforcing integrability in shape from shading algorithms. IEEE Transactions in Pattern Recognition and Machine Intelligence 10, 439 451 (1988)

10. Sim, T., Kanade, T.: Combining models and exemplars for face recognition: An illuminating example. In: Proceedings of the CVPR 2001 Workshop on Models versus Exemplars in Computer Vision (December 2001) 
11. Smith, W., Hancock, E.R.: Recovering facial shape and albedo using a statistical model of surface normal direction. In: Proc. ICCV, pp. 588-595 (2005)

12. Turk, M., Pentland, A.: Face recognition using eigenfaces. In: IEEE Conference on Computer Vision and Pattern Recognition (1991)

13. Worthington, P.L., Hancock, E.R.: New constraints on data-closeness and needle map consistency for shape-from-shaping. IEEE Transactions on Pattern Analysis and Machine Intelligence 21(12), 1250-1267 (1999) 